\title{
Cloud mass function in a gas rich dwarf galaxy
}

\author{
Takayuki Saitou and Asao Habe \\ Division of Physics, Graduate School of Science, Hokkaido University, \\ Sapporo 060-0810, Japan
}

Takashi Okamoto

Yukawa Institute for Theoretical Physics, Kyoto University, Kyoto 606-8502, Japan

\begin{abstract}
We study cloud mass function (here after CMF) obtained in a Grape-SPH simulation of model galaxy. The CMF can be fitted by a simple power law, and its power law index is $\sim-2.0$, which closes to the observational results.
\end{abstract}

\section{Introduction}

Observed CMF shows simple power law distribution,

$$
d N / d M \propto M^{\alpha},
$$

and $\alpha=-1.0$ to -2.0 over about 10 orders of magnitude in mass(e.g. Blitz 1993, Fukui et al 1999). It is interesting that how the function form of CMF is decided by interstellar processes (e.g. cloud-cloud collision, star formation \& feedback mechanism, and other physical mechanisms). CMF may relate with star formation and cloud evolution. Hence, what is an origin of this power law relation is an important question.

\section{Method}

We calculate dynamical evolution of interstellar medium by Grape-SPH (Steinmetz 1996, Koda et al 1999, etc), and considering thermal evolution of selfgravitational fluid including radiative cooling and FUV heating, excepting for star formation and feedback for simplicity.

Our model galaxy has an isothermal halo potential, an exponential stellar $\operatorname{disk}\left(3 \times 10^{9} M_{\odot} / 4096\right.$ particles $)$ with gas $\left(3 \times 10^{8} M_{\odot} / 65536\right.$ particles $)$.

\subsection{Result}

To find massive clouds in our simulation, we use friends-of-friends algorithm for low temperature $(T \leq 8000 \mathrm{~K})$ particles. Typically, we can pick up 300 clumps by the method. CMF in our result is Figure 1. The power law index $\alpha \sim-2.0$, which does not change after $200 \mathrm{Myr}$. 


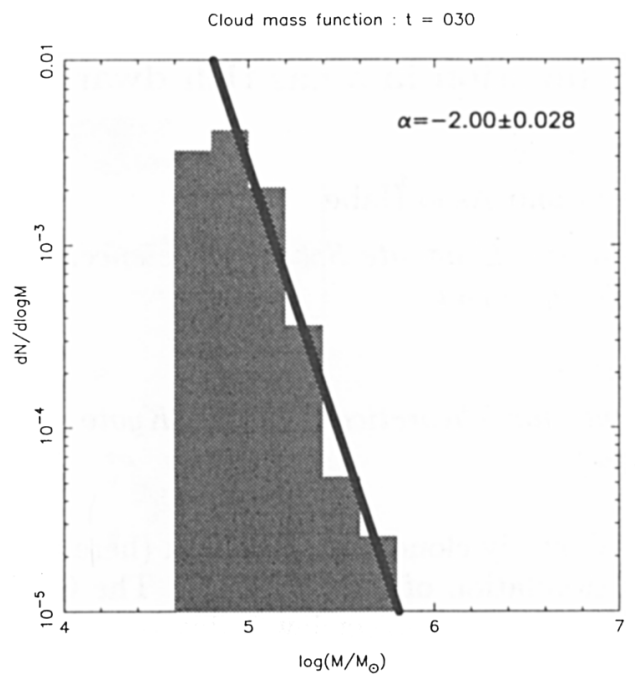

Figure 1. Cloud mass function at 300Myr. Least-square fits to our CMF give $\alpha \sim-2.0$.

\section{Summary \& Discussion}

We investigate galactic cloud mass function with a Grape-SPH simulation of model galaxy. Our simple model can reproduce observed molecular clouds mass function.

In our simulation, CMF has a power law form as a result of merging of smaller clouds, but their collision frequencies is not so large for CMF to attain steady state.

We will consider star formation \& feedback, since these very important for interstellar gas dynamics.

\section{References}

Blitz, L. 1996, in Protstars and Planests III, ed. E.H. Levy \& J.I. Lunine (Tucson:Univ. Arizona Press), 125

Fukui, Y. et al 1999, PASJ, 51, 745

Koda, J., Sofue, Y. \& Wada, K. 1999, ApJ, 532, 214

Steinmetz, M. 1996, MNRAS, 278, 1005 